\title{
Soft X-ray backlighter source driven by a short-pulse laser for pump-probe characterization of warm dense matter
}

Cite as: Rev. Sci. Instrum. 89, 10F122 (2018); https://doi.org/10.1063/1.5039419

Submitted: 08 May 2018 . Accepted: 29 June 2018 . Published Online: 16 October 2018

C. McGuffey (D), M. Dozières (D), J. Kim (D), A. Savin (D), J. Park (D), J. Emig, C. Brabetz (D), L. Carlson, R. F. Heeter (D), H. S. McLean (D), J. Moody, M. B. Schneider (D), M. S. Wei, and F. N. Beg

\section{COLLECTIONS}

Paper published as part of the special topic on Proceedings of the 22nd Topical Conference on High-Temperature Plasma Diagnostics

Note: Paper published as part of the Proceedings of the 22nd Topical Conference on High-Temperature Plasma Diagnostics, San Diego, California, April 2018.
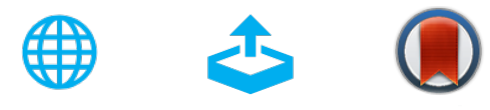

View Online

Export Citation

\section{ARTICLES YOU MAY BE INTERESTED IN}

High-pressure chemistry of hydrocarbons relevant to planetary interiors and inertial confinement fusion

Physics of Plasmas 25, 056313 (2018); https://doi.org/10.1063/1.5017908

X-ray spectrometer throughput model for (selected) flat Bragg crystal spectrometers on laser plasma facilities

Review of Scientific Instruments 89, 10 F119 (2018); https://doi.org/10.1063/1.5039423

A new toroidal x-ray crystal spectrometer for the diagnosis of high energy density plasmas at the National Ignition Facility

Review of Scientific Instruments 89, 10 F118 (2018); https://doi.org/10.1063/1.5036806

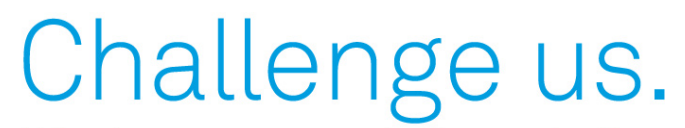

What are your needs for periodic

signal detection?

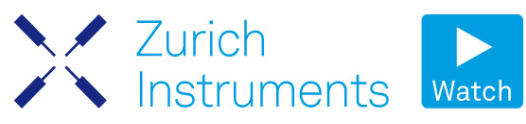

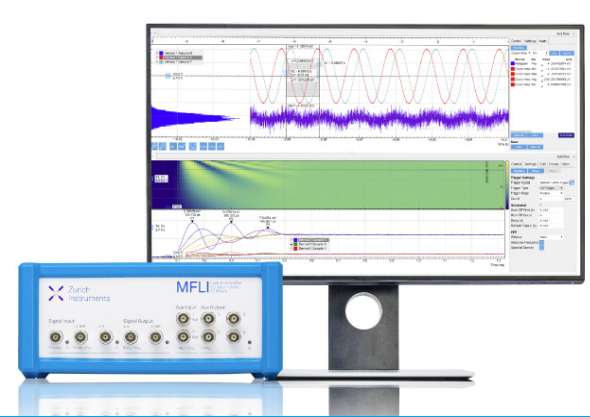

89, $10 \mathrm{~F} 122$ 


\title{
Soft X-ray backlighter source driven by a short-pulse laser for pump-probe characterization of warm dense matter
}

\author{
C. McGuffey, ${ }^{1, a)}$ M. Dozières, ${ }^{1}$ J. Kim, ${ }^{1}$ A. Savin, ${ }^{2}$ J. Park, ${ }^{3}$ J. Emig, ${ }^{3}$ C. Brabetz, ${ }^{4}$ \\ L. Carlson, ${ }^{5}$ R. F. Heeter, ${ }^{3}$ H. S. McLean, ${ }^{3}$ J. Moody, ${ }^{3}$ M. B. Schneider, ${ }^{3}$ M. S. Wei, ${ }^{5}$ \\ and F. N. Beg ${ }^{1}$ \\ ${ }^{1}$ Center for Energy Research, University of California, San Diego, La Jolla, California 92093, USA \\ ${ }^{2}$ Clarendon Laboratory, Department of Physics, University of Oxford, Oxford OX1 3PU, United Kingdom \\ ${ }^{3}$ Lawrence Livermore National Laboratory, Livermore, California 94550, USA \\ ${ }^{4}$ Plasma Physics Department, GSI Helmholtzzentrum, Darmstadt D-64291, Germany \\ ${ }^{5}$ General Atomics, San Diego, California 92121, USA
}

(Presented 18 April 2018; received 8 May 2018; accepted 29 June 2018; published online 16 October 2018)

\begin{abstract}
Here we propose a pump-probe X-ray absorption spectroscopy temperature measurement technique appropriate for matter having temperature in the range of 10 to a few $100 \mathrm{eV}$ and density up to solid density. Atomic modeling simulations indicate that for various low- to mid-Z materials in this range the energy and optical depth of bound-bound and bound-free absorption features are sensitive to temperature. We discuss sample thickness and tamp layer considerations. A series of experimental investigations was carried out using a range of laser parameters with pulse duration $\leq 5 \mathrm{ps}$ and various pure and alloyed materials to identify backlighter sources suitable for the technique. Published by AIP Publishing. https://doi.org/10.1063/1.5039419
\end{abstract}

\section{CONCEPTUAL DEVELOPMENT FOR A PUMP-PROBE WARM TEMPERATURE DIAGNOSTIC}

\section{A. X-ray absorption spectroscopy in warm systems}

$\mathrm{X}$-ray absorption spectroscopy is a general term for techniques applied to characterize a material sample based on its absorption features due to bound electrons. X-rays from a source backlight a sample and are spectrally resolved. Among the well-known techniques are X-ray Absorption Near Edge Structure (XANES) and Extended X-ray Absorption Fine Structure (EXAFS). Both may be applied to determine the chemical properties of a compound or crystalline structure, ${ }^{1}$ usually for a sample at standard temperature and pressure or in a compressed state. ${ }^{2-4}$

Absorption spectroscopy can be extended to transient plasma samples in high energy-density physics experiments such as an X-ray irradiated foil ${ }^{5}$ or an X-ray imploded liner. ${ }^{6}$ These targets may be pushed into the warm, dense matter (WDM) regime (temperature, $\mathrm{T} \sim 1-100 \mathrm{eV}$; density, $\rho \sim 0.01$ $10 \mathrm{~g} / \mathrm{cc}$ ). The state properties of WDM are notorious for being difficult to model with either plasma or solid state analytical frameworks, yet they must be accurately known to make planetary core models ${ }^{7}$ and to simulate ablator and shell behavior in inertial confinement fusion implosions. ${ }^{8}$ WDM conditions can be reached by irradiating an initially solid target with high power so that it undergoes a rapid increase in temperature and relatively prolonged expansion.

Note: Paper published as part of the Proceedings of the 22nd Topical Conference on High-Temperature Plasma Diagnostics, San Diego, California, April 2018.

a) Author to whom correspondence should be addressed: cmcguffey@ ucsd.edu.

\section{B. Motivation for a short pulse, low $Z$ backlighter}

The transient nature of WDM samples created in the lab presents a challenge to characterize the sample in the appropriate time scale. For example, matter can be heated on a time scale of picoseconds by an ultrafast laser or tens of ps by a laser-driven proton beam, ${ }^{9,10}$ motivating the development of ps-scale time-resolved measurement techniques.

A conventional backlighter is a metal foil irradiated by a ns-duration laser pulse. Metals with a high atomic number, $\mathrm{Z}$, can make broadband backlighters using M-band emission, but classification guidelines may restrict the choice of backlighter. A major drawback of using a ns backlighter for absorption spectroscopy of a transient sample is the necessity to streak the measurement, which can add practical difficulty.

In this work, we layout an absorption spectroscopy conceptual design using a short pulse backlighter and present $\mathrm{X}$-ray measurements using a range of laser parameters with pulse duration $\leq 5 \mathrm{ps}$ and various pure and alloyed materials. Even with the short laser duration, the backlighter emission may last a few tens of ps. ${ }^{11}$ The technique centers on changes to the $\mathrm{K}_{\mathrm{I}}$ edge or $\mathrm{L}_{\mathrm{II} / \mathrm{III}}$ edge absorption features as a sample is heated to a high degree of ionization. A similar technique was demonstrated with $\mathrm{Al}$ heated directly by a 300 fs laser. ${ }^{12}$ We propose applying the technique such that the sample is heated by a proton beam from a short pulse laser incident on a foil in the normal sheath acceleration (TNSA) mechanism regime. The proton beam would have a broad spectrum extending up to tens of $\mathrm{MeV}$, could be focused to $<100 \mu \mathrm{m}$ using a curved target as in the cited work by Patel, ${ }^{9}$ and stretched temporally to many tens of ps by choosing a standoff distance of $\sim 500 \mu \mathrm{m}$. 


\section{Modeling temperature-sensitive edge features}

The PrismSPECT atomic physics code ${ }^{13}$ is used to calculate steady state opacities in plasma using local thermodynamic equilibrium (LTE) collisional and radiative transition rates. All plotted examples here include convolution with a resolving power $\mathrm{E} / \Delta \mathrm{E}=300$. Example transmission curves are shown in Fig. 1 for the Al K-edge and $\mathrm{Cu}$ L-edge. The cold, simple, familiar edges (black curves) become much more complex with a plasma temperature of $20 \mathrm{eV}$. In particular, single edge transitions are turned into multi-stepped edges due to the presence of multiple charge states, and multiple bound-bound transitions with energy less than the cold edge appear due to upper state vacancies.

Temperature may be inferred from experimental data by fitting absorption spectra to a tightly resolved series of calculations with temperature and density parameters. For $10 \mathrm{eV}$, calculations with $\mathrm{Al}$ show no change in the absorption structure, but only a slight lowering of the K-edge energy, presumably due to the continuum lowering ${ }^{14}$ model used. At $15 \mathrm{eV}$, a strong bound-bound transition emerges at $1492 \mathrm{eV}$, easily distinguishable from the edge, and at $22 \mathrm{eV}$, a second bound-bound transition emerges at $1500 \mathrm{eV}$. Above this temperature, the edge structure is temperature-sensitive. These features are deep and resolvable, giving hope that experiment data could be fit to them given a backlighter spectrum that is flat, constant during the probing time, and reproducible.

In designing an experiment suitable to apply this principle, it is important to consider the temperature expected to be reached and choose a material whose energy levels will be significantly modified by such a temperature. Taking the series of calculations near the $\mathrm{Cu} \mathrm{L}$-edge, for example, one sees that at $50 \mathrm{eV}$ temperature, numerous features spanning $100 \mathrm{eV}$ are available to fit. This roughly corresponds to the
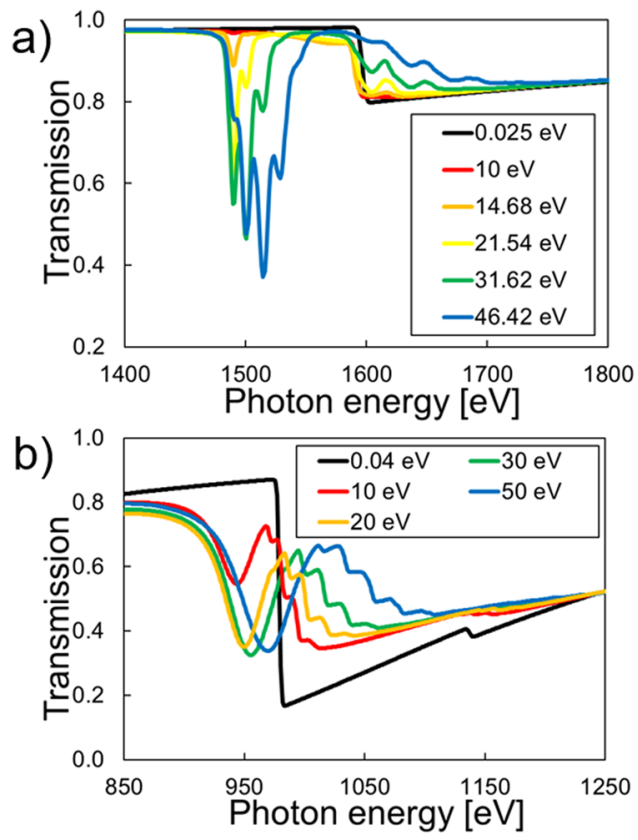

FIG. 1. Calculated X-ray transmission around the Al K-edge (a) and the $\mathrm{Cu}$ L edges (b) for solid-density samples with temperatures up to $50 \mathrm{eV}$. temperature where the $\mathrm{Cu}$ ions reach the stable Ar-like configuration (exceeds $1 \%$ abundance), and five different ion states have $>10 \%$ abundance (K-like through V-like). The technique could be extended to higher temperature by choosing a material with a higher edge energy; for example, at several hundred $\mathrm{eV}$, the $\mathrm{Cl}$, $\mathrm{K}$, or Ti $\mathrm{K}$-edge would be appropriate.

A practical consideration for making transmission measurements is that the inverse of transmission must be less than the ratio of signal to noise, $\mathrm{S} / \mathrm{N}$, so that even the darkest features can be distinguished from background. This leads to a constraint on the areal density of the sample. As a starting point, various thicknesses with uniform solid density are considered. Figure 2 shows again the calculated cold and hot transmission spectrum around the $\mathrm{Al} \mathrm{K}$-edge, this time for solid $\mathrm{Al}$ of various thicknesses. A thickness between 0.1 and $0.2 \mu \mathrm{m}$ would be appropriate given a $\mathrm{S} / \mathrm{N}>5$. Thickness $\geq 0.5 \mu \mathrm{m}$ would be too thick.

Another consideration is that the target can expand during the experiment. The calculations above show that the optimal foil thickness in the probing dimension is sub-micron, yet this means that an expansion rate of just $10^{-4} \mathrm{c}(30 \mathrm{~nm} / \mathrm{ps})$ along the probing dimension can quickly cause the density to decrease. For heating with a TNSA-produced proton beam, the target may even be preheated by X-rays and fast electrons, causing expansion prior to the arrival of the protons. In such circumstances, a tamp could be applied to slow this expansion. The need for a tamp with inertia competes with the need for it to have low opacity. Even more important than opacity, the tamp must have a low atomic number, $\mathrm{Z}$, so that no absorption edges could obfuscate the absorption of the sample. For a $100 \mathrm{~nm}$ thick $\mathrm{Cu}$ foil sample, appropriate tamp choices might be $1 \mu \mathrm{m}$ polypropylene/parylene-N or $0.5 \mu \mathrm{m} \mathrm{Be}$, which have roughly the same areal mass as the sample and $>75 \%$ transmission for photon energy, hv, $>1 \mathrm{keV}$.

The backlighter was an oval shaped foil with minor and major width of $0.300 \mathrm{~mm} \times 1.00 \mathrm{~mm}$, respectively, and typically $25 \mu \mathrm{m}$ thickness. The minor width was chosen to ensure precise positioning between the X-ray source and the probe region. The sample was a $2 \times 1 \mathrm{~mm}$ foil of either $100 \mathrm{~nm} \mathrm{Cu}$ or $200 \mathrm{~nm} \mathrm{Al}$ with $500 \mathrm{~nm}$ parylene-N tamp on both sides produced by Luxel Corporation. On both sides of this triple foil, $125 \mu \mathrm{m}$ thick Kapton was adhered to provide structure. The Kapton layer had a $250 \times 250 \mu \mathrm{m}$ window to restrict the probed region. Examples are shown in Fig. 3.

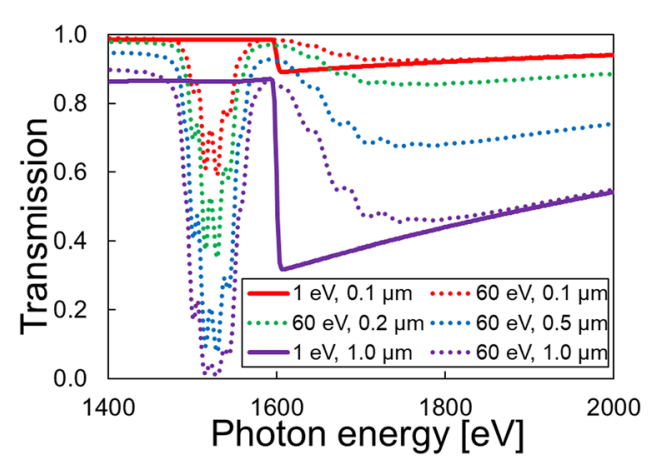

FIG. 2. Calculated transmission for various thicknesses of solid Al for a temperature of 1 or $60 \mathrm{eV}$. 


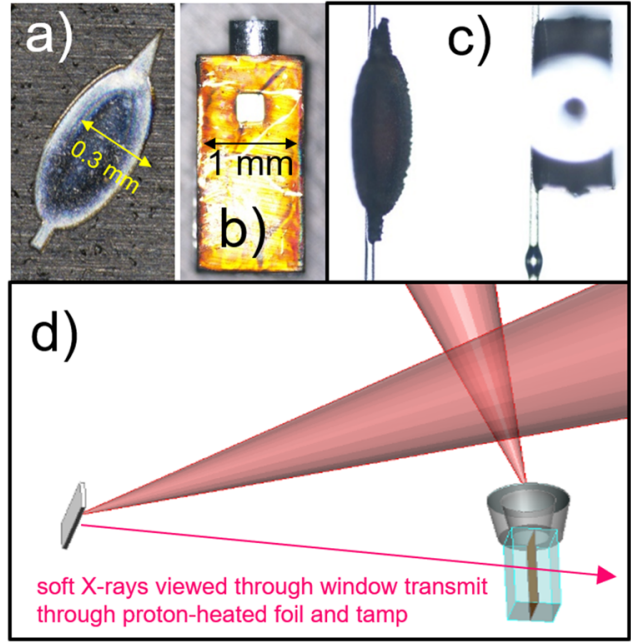

FIG. 3. (a) A laser-cut backlighter target. (b) A sample package viewed faceon with a curved foil for proton generation is on top. (c) A mounted target pair including a backlighter and sample package viewed end-on. The backlighter is precisely in the plane of the window; the curved foil is foreground. (d) Schematic of a proton-heating configuration.

\section{X-RAY EMISSION MEASUREMENTS FROM SHORT-PULSE LASER-DRIVEN BACKLIGHTER FOILS}

In designing a successful absorption experiment, it is important to characterize the backlighter emission. Emission from a backlighter driven by a short pulse laser, in particular, needs to be tested. Backlighter testing was carried out with five sets of laser parameters at three laser facilities as a prerequisite to absorption experiments. The laser parameters are summarized in Table I. In each case, two spectrometers viewed the backlighter target: a HOPG-based spectrometer, referred to as DCHOPG,${ }^{15}$ with resolving power $\mathrm{E} / \Delta \mathrm{E} \approx 100$, measuring the range 7.5-10 keV, typical of $\mathrm{K}$-shell emission for the first row of transition metals, and a soft $\mathrm{X}$-ray variable spacing grating (VSG) spectrometer, ${ }^{16,17}$ based on a curved grating, measuring the range $200-1800 \mathrm{eV}$ with $\mathrm{E} / \Delta \mathrm{E} \approx 300$. Both spectrometers had aluminized mylar filters directly in front of imaging plate detectors. Only backlighter emission data will be presented here, not absorption measurements.

\section{A. Identifying appropriate backlighter target material}

At the COMET laser in the Jupiter Laser Facility (JLF) ${ }^{18}$ at Lawrence Livermore National Laboratory, several backlighter materials were fielded, including $\mathrm{Al}, \mathrm{Ni}, \mathrm{Zn}$, and $\mathrm{Ge}$ pure metals as well as alloys brass (Cu 63\%, Zn 37\%), Kovar (Fe 54\%, Ni 29\%, Co 17\%), and Hastelloy c276 (Ni 57\%,

TABLE I. Laser parameters. All lasers operated at $1.05 \mu \mathrm{m}$ wavelength.

\begin{tabular}{lcccc}
\hline \hline Laser & Energy $(\mathrm{J})$ & Duration $(\mathrm{ps})$ & Spot $(\mu \mathrm{m})$ & Intensity $\left(\mathrm{W} / \mathrm{cm}^{2}\right)$ \\
\hline COMET A & 8 & 0.7 & 12 & $8.0 \times 10^{18}$ \\
COMET B & 8 & 0.7 & $>60$ & $<5.0 \times 10^{17}$ \\
PHELIX & 25 & 0.5 & 25 & $8.0 \times 10^{18}$ \\
TITAN A & 12 & 0.7 & 12 & $1.3 \times 10^{19}$ \\
TITAN B & 25 & 5.0 & 12 & $3.5 \times 10^{18}$ \\
\hline \hline
\end{tabular}

Mo $17 \%$, Cr $16 \%$, Fe $4 \%-7 \%$, W 3\%-5\%, Co <2.5\%). The laser irradiated the backlighter either with best focus or with a defocused beam to reduce the intensity so as to eliminate relativistic electrons from the interaction.

Figure 4 shows the energy calibration (a) of the VSG and emission data before filter correction. Energy calibration was achieved by identifying the $\mathrm{O}$ edge and $\mathrm{C}$ edge (not plotted) from the filter and emission lines from a shot on a glass target. In Fig. 4(b), it is seen that emission in the VSG spectral window is dominated by line emissions attributable to Balmer-like transitions of hot ions of the target element. The emission at the low energy side of the plot is due primarily to 2 nd order collection. In Fig. 4(c), the jagged Ni spectrum is plotted alongside
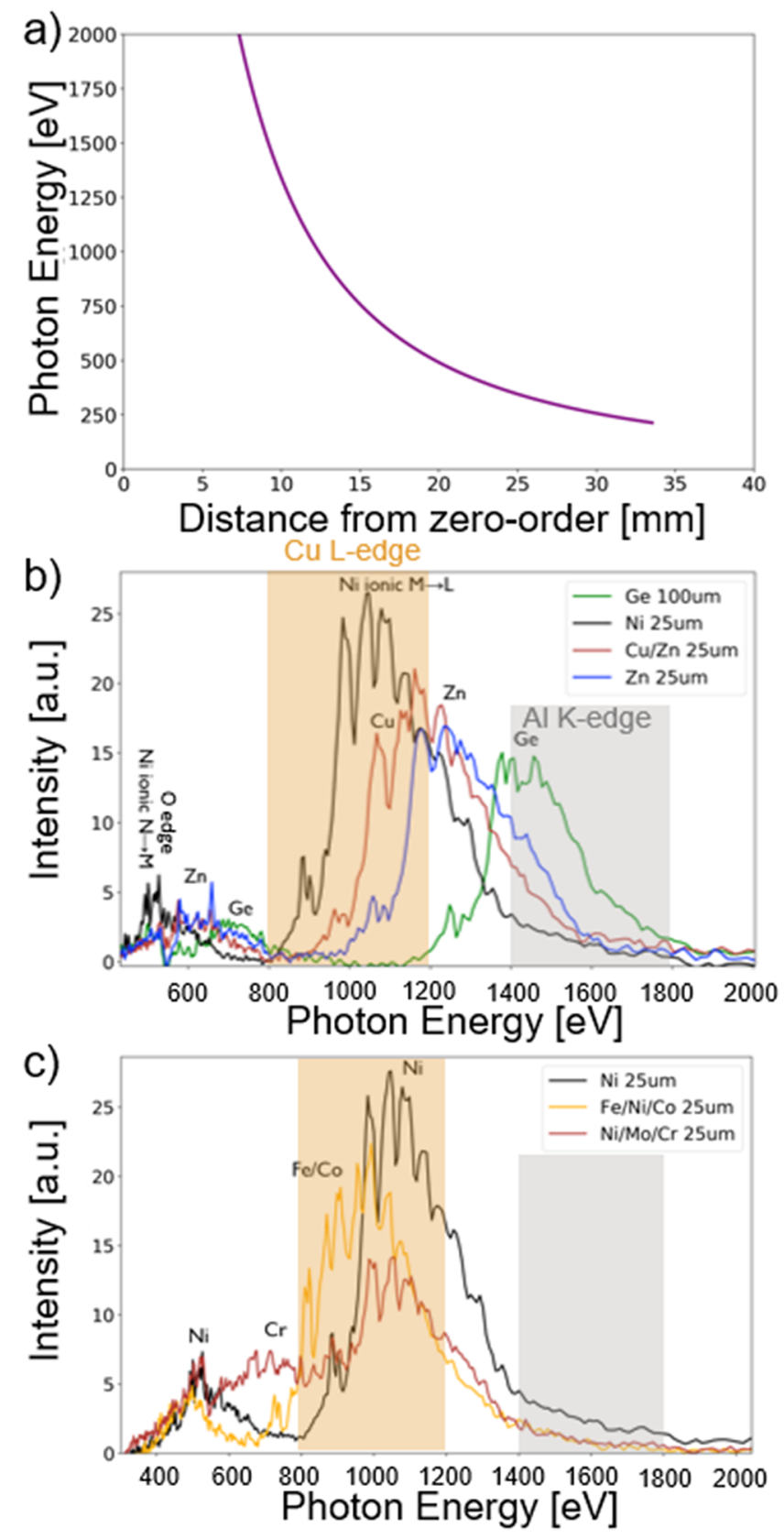

FIG. 4. (a) Energy calibration of the VSG. (b) Raw VSG spectra from various targets. (c) The spectra from two alloys are presented for comparison to pure $\mathrm{Ni}$. The spectral range of interest for absorption spectroscopy of the Cu L-edge and $\mathrm{Al} \mathrm{K}$-edge is shown by the tan and gray bands, respectively. 
two alloys of interest. Kovar, containing Fe and Co, the two elements below $\mathrm{Ni}$, produces a spectrum downshifted from $\mathrm{Ni}$ and better overlapped with the band of interest for absorption spectroscopy of the $\mathrm{Cu}$ L-edge. Hastelloy, with contributions from $\mathrm{Cr}, \mathrm{Fe}$, and $\mathrm{Co}$, synthesizes an overall much wider, smoother spectrum than pure $\mathrm{Ni}$.

PrismSPECT was used to calculate the emission of a hot plasma representing the front surface plasma on the backlighter. In a series of simulations of $\mathrm{Ni}$ with parameters such as temperature and density, it was observed that the ionic lines in the output spectrum could be adjusted primarily by changing the temperature (up to $400 \mathrm{eV}$ ). A single simulation, however, could not reproduce the range of lines observed in the experiment. This should come as no surprise as a real backlighter emission source would have a spatially varying and time-varying plasma temperature. Figure 5 shows how the experimental spectrum can be reproduced by adding piecewise contributions representing different regions of the backlighter. For Ni [Fig. 5(a)], from the series of simulations, calculations with $\mathrm{Ni}$ at temperatures of 200 and $275 \mathrm{eV}$ were the best pair for reproducing the width of the hot $\mathrm{Ni}$ contribution. The ratio of the two contributions was treated as a free parameter, and the combined spectrum was normalized for comparison to the experimental data. For the Hastelloy target [Fig. 5(b)], the same two contributions from Ni were combined with calculations of $\mathrm{Cr}$ at the three temperatures to reproduce the overall shape of the dominant features of the experimental spectrum.
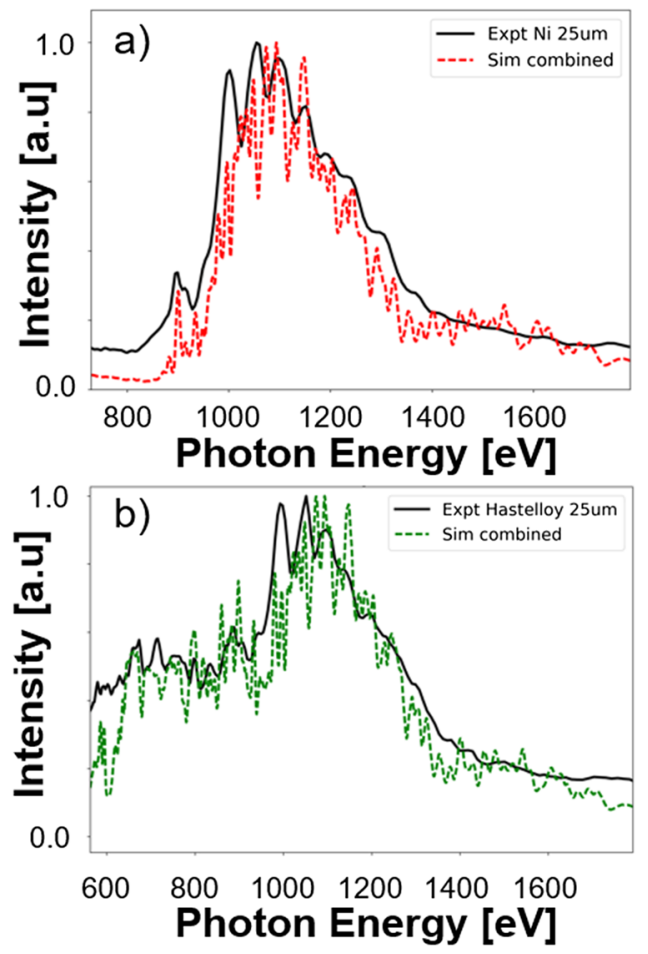

FIG. 5. Corrected experimental data are compared to PrismSPECT emission calculations convolved with the detector resolving power for pure $\mathrm{Ni}$ (a) and $\mathrm{Ni}$ alloy (b). The combined simulation curve in (a) includes the simulated emission from Ni at $0.1 \mathrm{~g} / \mathrm{cm}^{3}$ and two temperatures, $200 \mathrm{eV}$ and $275 \mathrm{eV}$. The combined simulation curve in (b) includes those same contributions as well as $\mathrm{Cr}$ at $0.1 \mathrm{~g} / \mathrm{cm}^{3}$ and three temperatures: $300 \mathrm{eV}, 250 \mathrm{eV}$, and $125 \mathrm{eV}$.

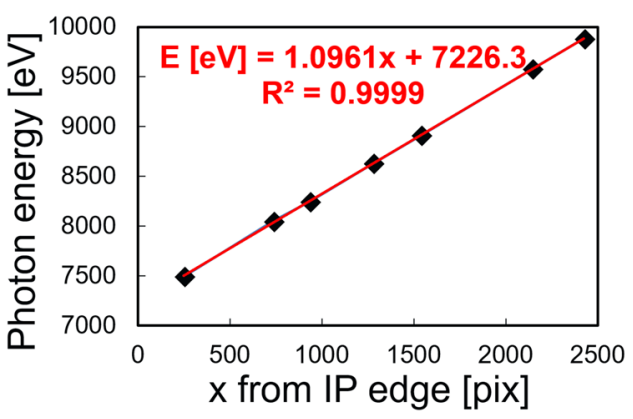

FIG. 6. DCHOPG energy calibration. Each pixel is $50 \mu \mathrm{m}$ along the Imaging Plate (IP).

The DCHOPG spectrometer recorded K shell emission from the backlighter targets. While the $\mathrm{X}$-ray energies are too high to be relevant to the X-ray absorption spectroscopy technique, we will see that the data give important understanding about the conditions in the backlighter target. The data typically showed only the $\mathrm{K} \alpha$ and $\mathrm{K} \beta$ of the target material(s). The Hastelloy target also showed Mo Ka in 2nd order. Figure 6 shows calibration of the spectrometer using the $\mathrm{K} \alpha$ and $\mathrm{K} \beta$ lines from $\mathrm{Ni}, \mathrm{Cu}$, and $\mathrm{Zn}$ as well as $\mathrm{K} \alpha$ from $\mathrm{Ge}$.

\section{B. Backlighter adequacy is highly dependent on laser parameters}

When the COMET laser was defocused, the emission intensity of the strongest lines measured with VSG dropped by roughly a factor of three, and the envelope of the emission changed, with the higher energy ionic lines disappearing. The signal in the K $\alpha$ peaks measured on DCHOPG decreased by a factor of $\geq 4$ for three target types and disappeared below the detection level in all other targets. Since the Ka emission is due to fast electron collisions with $\mathrm{K}$-shell electrons, the sharp decline in $\mathrm{K} \alpha$ emission for non-relativistic intensity is as expected.

On the PHELIX laser ${ }^{19}$ at the GSI Helmholtzzentrum für Schwerionenforschung, the Hastelloy backlighter was fielded with the same DCHOPG spectrometer with the same intensity as on COMET but with $4 \mathrm{X}$ higher laser energy and larger focal spot. The spectrum was similar to that on COMET, including $\mathrm{Ni} \mathrm{K} \alpha$, Ni K $\beta$, and Mo K $\alpha$ in 2nd order.

On the Titan laser, also at JLF, the Hastelloy backlighter target was fielded in one case (A) with similar laser parameters to COMET but 50\% higher energy and another case (B) with much longer pulse duration (5 ps). In case A, the DCHOPG shape (green curve on Fig. 7) was similar to COMET and PHELIX but with higher continuum, significantly a higher Mo $\mathrm{K} \alpha$ signal, and some contribution from the trace W. In case B (yellow curve on Fig. 7), however, the spectrum was rich with Li-like, He-like, and Lyman $\mathrm{Ni}$ ionic lines, indicating that the backlighter reached very high temperature. With condition Titan B, the VSG data were plagued by a strong background signal for all shots. The background was moved by adding a permanent magnet in front of the spectrometer, suggesting that the background originated from fast electrons inducing fluorescence inside. This made it impossible to record the soft X-ray spectrum for Titan B conditions. 


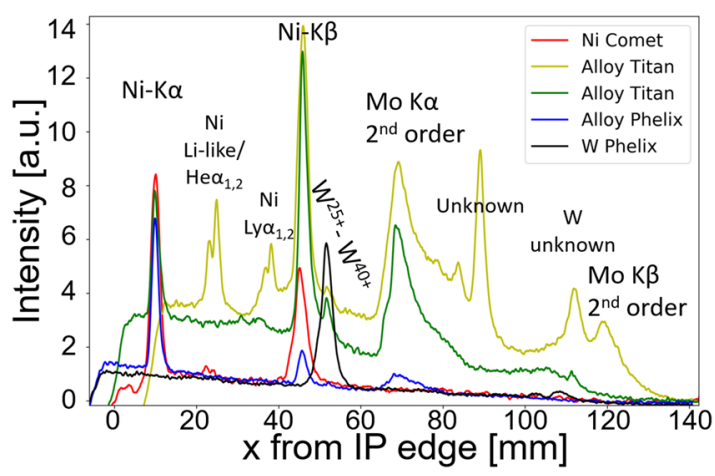

FIG. 7. DCHOPG emission shows only "cold" Ni and Mo lines with the subps COMET, PHELIX, and TITAN A drive. With the TITAN B 5 ps drive, hot ionic $\mathrm{Ni}$ and $\mathrm{W}$ lines emerge.

\section{CONCLUSIONS AND OUTLOOK}

In summary, a pump-probe technique and target pair design have been developed for determining the temperature of a warm, dense matter sample using X-ray absorption spectroscopy. This approach could bolster our ability to characterize WDM. The technique avoids the complications of an X-ray streak camera and can have temporal resolution as short as the emission lifetime of the backlighter. Using two picosecond-class lasers, the method could be applied to time-resolve isochoric heating of a sample by TNSA protons, which move much more slowly than the X-ray probe.

A short pulse laser driven backlighter, which is nonconventional, was explored. An appropriate low $\mathrm{Z}$ material, Hastelloy, was found which produced a somewhat broad spectrum needed for absorption spectroscopy. Other readily available candidate materials that could be appropriate backlighters for the $\mathrm{Cu}$ L-edge include Inconel $617(\mathrm{Ni}, \mathrm{Cr}, \mathrm{Co})$ and Nichrome $\mathrm{C}(\mathrm{Ni}, \mathrm{Fe}, \mathrm{Cr})$ which have significant abundance of three elements with hot lines in the appropriate range and a gap between, which helped give Hastelloy its broader spectrum.

When the pulse duration was sub-ps with intensity $>10^{18} \mathrm{~W} / \mathrm{cm}^{2}$, L-shell emission was brighter and broader due to an increased number of ionic states. This is desirable for the backlighter. When the intensity remained high and the pulse duration was increased to $5 \mathrm{ps}$, more ionic K-shell line emission was seen, but the increase in fast electron production led to a background originating in the diagnostic that blocked the backlighter signal. This gives guidance on the laser parameters that may be most suitable for short pulse backlighter absorption measurements.

\section{ACKNOWLEDGMENTS}

This work was performed under the auspices of the Department of Energy through the Fusion Energy Sciences HEDLP program under grant Award No. DE-SC0014600. A.S. acknowledges support from EPSRC Grant EP/N509711/1 and STFC Grant ST/P000967/1. We thank B. Stuart, B. Cauble, V. Bagnoud, P. Norreys, and the laser staff at the Jupiter and PHELIX Laser Facilities for devoted operational support, H. Reynolds, L. Gonzalez, J. Williams, and D. Kaczala of General Atomics for creative target fabrication efforts, as well as E. Magee and R. Shepherd for useful discussions.

${ }^{1}$ M. Wilke, F. Farges, P.-E. Petit, G. E. Brown, Jr., and F. Martin, Am. Mineral. 86, 714 (2001).

${ }^{2}$ B. I. Cho et al., Phys. Rev. Lett. 106, 167601 (2011).

${ }^{3}$ B. I. Cho et al., Sci. Rep. 6, 18843 (2016).

${ }^{4}$ R. Torchio et al., Sci. Rep. 6, 26402 (2016).

${ }^{5}$ J. E. Bailey et al., Nature 517, 56 (2014).

${ }^{6}$ S. B. Hansen et al., High Energy Density Phys. 24, 39 (2017).

${ }^{7}$ N. Nettelmann, R. Redmer, and D. Blaschke, Phys. Part. Nucl. 39, 1122 (2008).

${ }^{8}$ D. E. Hanson, L. A. Collins, J. D. Kress, and M. P. Desjarlais, Phys. Plasmas 18, 082704 (2011).

${ }^{9}$ P. K. Patel, A. J. Mackinnon, M. H. Key, T. E. Cowan, M. E. Foord, M. Allen, D. F. Price, H. Ruhl, P. T. Springer, and R. Stephens, Phys. Rev. Lett. 91, 125004 (2003).

${ }^{10}$ G. M. Dyer et al., Phys. Rev. Lett. 101, 015002 (2008).

${ }^{11}$ E. V. Marley et al., High Energy Density Phys. 25, 15 (2017).

${ }^{12}$ P. Audebert et al., Phys. Rev. Lett. 94, 025004 (2005).

${ }^{13}$ J. J. MacFarlane, I. E. Golovkin, P. Wang, P. R. Woodruff, and N. A. Pereyra, High Energy Density Phys. 3, 181 (2007).

${ }^{14}$ L. B. Fletcher et al., Phys. Rev. Lett. 112, 145004 (2014).

${ }^{15}$ K. U. Akli et al., J. Instrum. 5, P07008 (2010).

${ }^{16}$ K. V. Cone et al., Rev. Sci. Instrum. 81, 10E318 (2010).

${ }^{17}$ J. Park et al., Rev. Sci. Instrum. 81, 10E319 (2010).

${ }^{18}$ B. Stuart et al., in Conference on Lasers and Electro-Optics/Quantum Electronics and Laser Science Conference and Photonic Applications Systems Technologies, Technical Digest (CD) (Optical Society of America, 2006).

${ }^{19}$ V. Bagnoud et al., Appl. Phys. B 100, 137 (2010). 